\title{
The 1s-State Analysis Applied to High-Angle, Annular Dark-Field Image Interpretation-When Can We Use It?
}

\author{
Geoffrey R. Anstis* \\ Department of Applied Physics, University of Technology, Sydney, P.O. Box 123, Broadway, NSW 2007, Australia
}

\begin{abstract}
A small probe centered on an atomic column excites the bound and unbound states of the two-dimensional projected potential of the column. It has been argued that, even when several states are excited, only the $1 s$ state is sufficiently localized to contribute a signal to the high-angle detector. This article shows that non-1s states do make a significant contribution for certain incident probe profiles. The contribution of the $1 s$ state to the thermal diffuse scattering is calculated directly. Sub-Ångstrom probes formed by $\mathrm{C}_{\mathrm{s}}$-corrected lenses excite predominantly the $1 s$ state and contributions from other states are not very large. For probes of lower resolution when non-1s states are important, the integrated electron intensity at the column provides a better estimate of image intensity.
\end{abstract}

Key words: high-resolution electron microscopy, scanning transmission electron microscopy, high-angle annular dark-field imaging, $1 \mathrm{~s}$ state, thermal diffuse scattering, multislice method, frozen phonon

\section{INTRODUCTION}

The use of a high-angle, annular dark-field (HAADF) detector in a scanning transmission electron microscope (STEM) has proved very productive in determining the atomic structure of many materials, particularly those made up of clearly defined columns of atoms. Most of the electrons that are scattered through an angle sufficient to reach the detector have exchanged energy with the vibrational modes of the material, that is, undergone thermal diffuse scattering (TDS), and to do this they must be close to a column as they pass through the sample (Howie, 1979; Wang \& Cowley, 1989; Pennycook \& Jesson, 1991).

One of the methods that has been used for computing the atomic-resolution images obtained with this imaging mode is to calculate the intensity of the image-forming electron at each column position and then to weight the sum of these intensities by a factor that depends on the vibrational parameters of the atoms and the acceptance angles of the detector. Recently Rafferty et al. (2001) have argued that this approach may be in error, particularly for columns of atoms of high atomic number. These authors state that it is the $1 s$ state component of the electron wave, to be defined below, that determines the HAADF image intensity and the non- $1 s$ state components, although significantly excited by the fast electron, do not make a major contribution to the image.

Received February 10, 2003; accepted May 7, 2003.

*E-mail: Geoff.Anstis@uts.edu.au
The description of the electron wave within the sample in terms of the $1 s$ state and the non- $1 s$ state components arises from using the channeling approach to electron scattering. The scattered wave is expressed in terms of the quantum mechanical eigenstates of the two-dimensional projected potentials of atomic columns. The eigenstates are either bound (negative energy) or unbound. The bound states are labeled as $1 s, 2 s, 2 p$, and so forth. The number of bound states depends on the depth of the projected potential and the energy of the incident electron.

The $1 s$ state makes the major contribution to the HAADF image intensity of the column because an electron undergoes significant thermal diffuse scattering only if it passes close to the atomic column. The contribution of the other eigenstates is less because they are not as strongly peaked at the column as the $1 s$ state.

The conclusion of Rafferty et al. (2001) that image intensities calculated from the total intensity at the column may be in error are based on calculations that do not explicitly calculate the thermal diffuse scattering. For this reason, their study could be regarded as incomplete. This article calculates the thermal diffuse scattering explicitly and uses this calculated intensity to calculate the image intensity. It is found that images formed with TDS electrons may contain significant contributions from non-1s state electrons. Thus the conclusions of Rafferty et al. have limited validity. This article explores conditions under which their approach does provide a useful estimate of the image intensity.

We consider images of [110] InAs and show that the $1 s$ state is useful for a qualitative understanding of the image 
intensity under several imaging conditions. Only in some circumstances is it useful for quantitative predictions. For instance, it is a good approximation for determining images formed with a sub-Ångstrom probe that is focused on the entrance surface of the sample.

\section{Methods of Calculating HAAdf IMAGE INTENSITY}

Two approaches are employed to calculate the thermal diffuse scattering. Both are based on the multislice method of Cowley and Moodie (Cowley, 1995). The first may be considered as an extension of the work of Cowley (1988), who calculated TDS in a first-order approximation through use of a thermal scattering function that depends on the first derivative of the atomic potential. The present work includes higher-order scattering functions involving higherorder spatial derivatives and is described in more detail by Anstis (2001). It has been applied by Anderson et al. (1997) to estimate the $\mathrm{Al}$ concentration in $\mathrm{Al}_{x} \mathrm{Ga}_{1-x} \mathrm{As}$ from atomic resolution HAADF images. Equivalent schemes have been described by Dinges et al. (1995) and by Wang (1995).

The method requires that the elastic wave function be calculated at each atom and the amount of scattering of order $(s, t)$ to position $\left(U_{x}, U_{y}\right)$ in the reciprocal space plane perpendicular to the columns is determined by the function

$$
\begin{array}{r}
T_{s, t}(\mathbf{U})=\frac{(B / 2)^{t / 2}}{\sqrt{s !(t-s) !}} U_{x}^{s} U_{y}^{t-s} \Phi(\mathbf{U}) \exp \left(-\frac{1}{4} B U^{2}\right) \\
s \leq t=1,2,3, \ldots
\end{array}
$$

$U$ is a vector in reciprocal space. $\Phi(U) \exp \left(-\frac{1}{4} B U^{2}\right)$ is the atomic scattering factor for elastic scattering that, in the high-energy limit, is the Fourier transform of the configurational average of $\exp [i \sigma \bar{\varphi}(\mathbf{R})]$ where $\bar{\varphi}(\mathbf{R})$ is the projected atomic potential and $\sigma=2 \pi m e \lambda / h^{2}$ (Cowley, 1995). $\Phi(\mathrm{U})$ is a complex function with an imaginary part corresponding to the rate of thermal scattering (Cowley, 1995; Anstis, 1996). The average over all positions of an atom introduces $B$, the temperature parameter, which is proportional to the mean square amplitude of atomic vibration. The parameter $t$ is the order of the thermal scattering. $t=1$ corresponds to single thermal scattering. $t=2$ corresponds to thermal scattering of already thermally scattered electrons, and so on. The parameter $s$ is related to the amount of thermal scattering of order $t$ that is in the $x$ direction.

The other method of calculating the TDS is based on a Monte Carlo approach. We calculate the scattering by configurations of atoms at particular instances of time and find the average value of the total intensity of electrons scattered within the angular range defined by the detector to obtain an estimate of the image intensity. This method has been called the frozen phonon method and has been used exten- sively by Silcox's group (Loane et al., 1992). The deviations of atoms from their equilibrium positions are selected by using a random number generator. The mean square deviation of each atom species depends on its temperature parameter $B$. This approach requires the input of the elastic scattering function only.

To find the $1 s$ state associated with a column, the multislice method is modified so that the $z$ direction, the direction along the column, becomes an imaginary number. This change results in the projected potential of the column being an absorptive one. After the "wave" has propagated sufficiently far along the column, only the lowest-energy eigenstate component of the incident wave has significant intensity; all other components having been absorbed. Details are given by Anstis et al. (2003).

A probe in the form of a $1 s$ state centered on a column will propagate along a nonabsorbing atomic column without changing its shape. However if thermal scattering takes place, the amplitude of the wave function for the elastically scattered electron diminishes and its shape changes slightly as it propagates along the column.

The probe is defined by the reciprocal space expression

$$
P(U)=A(U) \exp \left[-i \pi\left(\lambda \varepsilon U^{2}+\frac{1}{2} \lambda^{3} C_{s} U^{4}\right)\right],
$$

where the aperture function $A(U)=1$ if $U<U_{\max }=$ $\sin \theta_{\max } / \lambda$ and is 0 otherwise. $C_{s}$ is the coefficient of spherical aberration and $\varepsilon$ is the amount of defocus.

The real space function $p(\mathbf{R})$ describing the probe is found by inverse Fourier transformation of $P(U)$. The probe can be expressed in terms of the $1 s$ state component and the non-1s state components

$$
p(\mathbf{R})=c_{1 s} \varphi_{1 s}(\mathbf{R})+c_{n} \varphi_{n}(\mathbf{R}, 0) .
$$

The wave function for the elastically scattered electron at distance $z$ along a column is given by

$$
\begin{aligned}
\Psi(\mathbf{R}, z)= & c_{1 s} \varphi_{1 s}(\mathbf{R}) \exp \left(-\mu_{1 s} z\right) \\
& \times \exp \left(-i \sigma E_{1 s} z\right)+c_{n} \varphi_{n}(\mathbf{R}, z),
\end{aligned}
$$

where $E_{1 s}$ is the energy of the $1 s$ state. $\mu_{1 s}$ is the absorption parameter for the $1 s$ state.

If the non-1s-state components have energies near zero, it may be a good approximation, for small values of $z$, to write

$$
\begin{aligned}
\Psi(\mathbf{R}, z)= & c_{1 s} \varphi_{1 s}(\mathbf{R}) \exp \left(-\mu_{1 s} z\right) \exp \left(-i \sigma E_{1 s} z\right) \\
& +c_{n} \varphi_{n}(\mathbf{R}, 0) \exp \left(-\mu_{n} z\right)
\end{aligned}
$$

where $\mu_{n}$ is the absorption parameter for the non- $1 s$ states. In this case, the intensity of the wave along the column peaks at regular intervals of length $2 \pi / \sigma E_{1 s}$. 

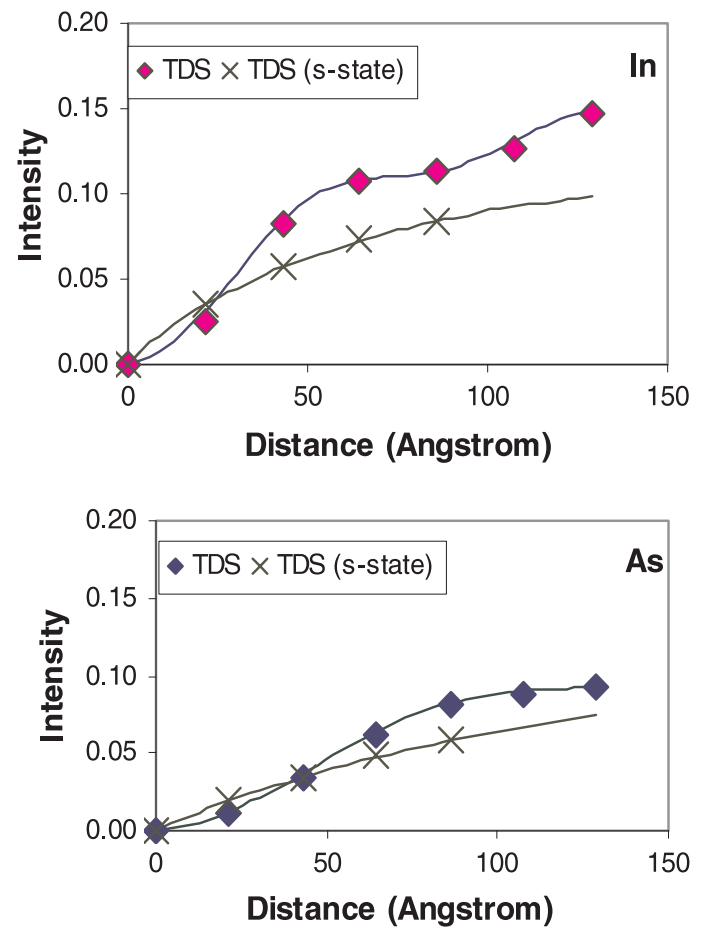

Figure 1. Intensity of electrons reaching HAADF detector as a function of column length for a probe centered on indium and arsenic columns. TDS is calculated from the full wave function and from its $s$-state component. The continuous lines are the scaled integrated intensities of elastically scattered electrons along the columns. The probe parameters are: $\alpha=9.7 \mathrm{mrad}, C_{s}=1 \mathrm{~mm}$, $\varepsilon=-44 \mathrm{~nm}$. Scaling factors are given in Table 1 .

The $1 s$ state is strongly peaked at the column center and generates a large amount of TDS. The other states are not so strongly concentrated and will generate less TDS. It is then reasonable to assume that for the purposes of calculating the TDS it is sufficient to consider only the $1 \mathrm{~s}$ state contribution. In the next section we consider several different probes and see what contribution the $1 s$ state makes to the HAADF image intensity.

\section{Computations of the CONTRiButions of $1 \mathrm{~s}$ States to HAADF Images}

The particular structure considered here is [110] InAs. Columns of In and As atoms are separated by $0.151 \mathrm{~nm}$ and the atoms along a column by $0.43 \mathrm{~nm}$. The temperature parameters are $0.002 \mathrm{~nm}^{2}$ for In and $0.004 \mathrm{~nm}^{2}$ for As. The unit cell of dimensions $0.606 \mathrm{~nm}$ by $0.43 \mathrm{~nm}$ is sampled at $256 \times 256$ points. The energy of incident electrons is $300 \mathrm{keV}$, at which the wavelength is $0.00197 \mathrm{~nm}$. The number of sampling points means that scattering out to 200 mrad is considered. The angles defining the HAADF detector are $60 \mathrm{mrad}$ and $200 \mathrm{mrad}$.
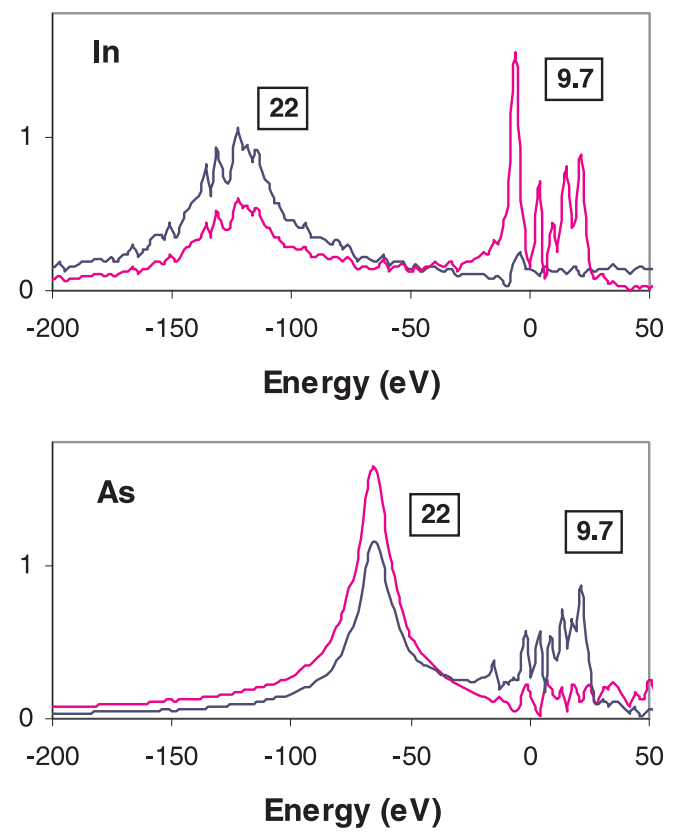

Figure 2. Distributions of energies of eigenstates excited by probes centered on atomic columns. Probe parameters are: $C_{s}=1 \mathrm{~mm}$, $\alpha=9.7 \mathrm{mrad}, \varepsilon=-44 \mathrm{~nm} ; C_{s}=0 \mathrm{~mm}, \alpha=22.4 \mathrm{mrad}, \varepsilon=0$.

First, consider a probe formed with an objective lens for which $C_{s}=1 \mathrm{~mm}, \varepsilon=-44 \mathrm{~nm}$, and $\alpha=9.74 \mathrm{mrad}$. Figure 1 shows how the HAADF intensity varies with distance along the column when the probe is placed on the indium column and on the gallium column. The continuous curves are the integrated elastic intensities of electrons on the columns that have been scaled to pass through the marked points that have been obtained by explicit calculation of the thermal diffuse scattering. The contributions to the signals from the $1 s$ states are also shown. Thus, at least in this case, it seems that the total electron intensity at the atom sites provides a good indication of the HAADF signal whereas the $1 s$ state accounts for only a fraction of the signal.

If the probe is formed with a $C_{s}$-corrected lens that is in-focus and with a semi-angle of convergence of 22.4 mrad, the signal does not exhibit a strong oscillation. Most of the signal is due to the $1 s$ state.

The oscillation of the In curve is due to the interference between the $1 s$ state and the other states. The distributions of eigenstates generated by these probe are shown in Figure 2. The distributions are determined by recording the wave functions at each column position as a function of distance $z$, and then Fourier transforming the set of values (see Anstis et al., 2003). There is a peak near energy -130 $\mathrm{eV}$ corresponding to the energy of the $1 s$ state. The strong peak near energy $-10 \mathrm{eV}$ that is excited for the lowerresolution probe corresponds to the excitation of the $2 \mathrm{~s}$ state of an indium column (Rafferty et al., 2001).

Figure 3 shows the signals for several imaging conditions that are scaled to the signal generated by the $1 s$ state. 

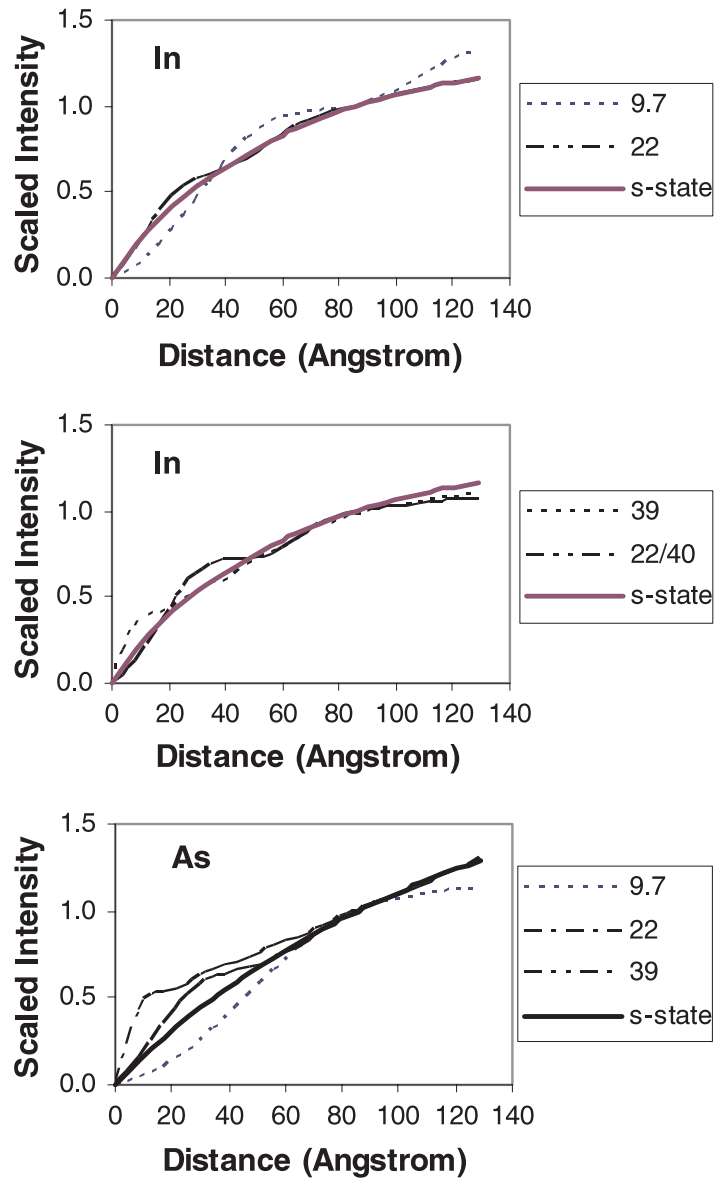

Figure 3. The intensity of electrons reaching the HAADF detector as a function of column length for In and As columns and for several probe parameters. The intensities have been scaled (see Table 1) to the integrated elastic intensity along the column due to an $s$-state probe. Curves are labeled according to the probe parameters. $C_{s}=1 \mathrm{~mm}, \alpha=9.7 \mathrm{mrad}, \varepsilon=-44 \mathrm{~nm} \mathrm{(9.7);} C_{s}=0 \mathrm{~mm}$, $\alpha=22.4 \mathrm{mrad}, \varepsilon=0$ (22) and $-4 \mathrm{~nm}(22 / 40) ; \alpha=39 \operatorname{mrad}, \varepsilon=0$ (39).

This figure shows that the $1 s$ state does provide an indication of the HAADF signal to be expected provided the scaling factor can be found. The scaling factors relating the intensity of the detected TDS generated by various probes and that generated by the $s$-state component of the probe are shown in Table 1. Also shown are the scaling factors relating the TDS to the total integrated elastic intensity of the probe along the column. The results indicate that the $s$-state scaling factor is the factor more sensitive to imaging conditions.

Figure 4 provides a measure of the contributions of the non- $1 s$ states to the HAADF signal. These contributions have been calculated by assuming the incident probe comprises all of the non-1s-state components of the probe formed by a lens with the indicated parameters. It is seen that a significant signal can be generated by a probe that does not have a $1 s$-state component.
Table 1. Scaling Factors to Obtain TDS by a 8.6-nm Column from: (1) TDS Generated by $s$-Wave Components of Probe; (2) the Integrated Elastic Intensity along the Column. The Probe Parameters Are Given.

\begin{tabular}{|c|c|c|c|c|}
\hline $\begin{array}{c}C_{s} \\
(\mathrm{~mm})\end{array}$ & $\begin{array}{c}\alpha \\
(\mathrm{mrad})\end{array}$ & $\begin{array}{c}\varepsilon \\
(\mathrm{nm})\end{array}$ & $\begin{array}{l}s \text {-state } \\
\text { scaling } \\
\text { factor }\end{array}$ & $\begin{array}{c}\text { Integrated } \\
\text { intensity } \\
\text { scaling } \\
\text { factor }\end{array}$ \\
\hline 1 & 9.7 & -44 & 1.4 & 0.9 \\
\hline 0 & 22.4 & 0 & 1.0 & 1.0 \\
\hline 0 & 22.4 & 4 & 1.8 & 0.9 \\
\hline 0 & 39.0 & 0 & 1.1 & 1.0 \\
\hline \multicolumn{3}{|c|}{$s$-state probe } & 1.0 & 1.0 \\
\hline
\end{tabular}

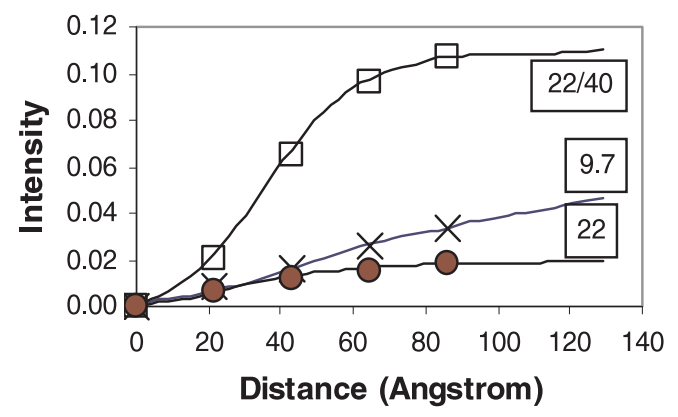

Figure 4. Contributions of non- $1 s$ states to the HAADF signal as a function of length of indium column for a variety of probe parameters (refer to Fig. 3).

\section{SUMMARY}

This article investigates the conjecture that only the $1 s$-state component of the scattered wave makes a significant contribution to the HAADF image intensity. It has been shown that this is the case for a probe of a shape similar to that of the $1 s$ state but that non- $1 s$ states are important for probes of other shapes. Knowing the intensity of the total electron wave at atomic sites can be used to obtain the HAADF image intensity with reasonable accuracy. Thus, there seems to be no reason to reexamine the many publications that have used this approach.

\section{ACKNOWLEDGMENT}

I thank Professor John Cowley for suggesting to me, many years ago, that eigenstates of atomic columns might be useful for analyzing high-resolution images. I regret not having undertaken the analysis sooner. 


\section{REFERENCES}

Anderson, S.C., Birkeland, C.R. \& Cockayne, D.J.H. (1997). An approach to quantitative compositional profiling at nearatomic resolution using high-angle annular dark field imaging. Ultramicroscopy 69, 83-103.

Anstis, G.R. (1996). Corrections to atomic scattering factors for high-energy electrons arising from atomic vibrations. Acta Cryst A 52, 450-455.

Anstis, G.R. (2001). Imaging point defects using a transmission electron microscope with controllable spherical aberration. Philos Mag B 81, 1687-1699.

Anstis, G.R., CaI, D.Q. \& Cockayne, D.J.H. (2003). Limitations on the s-state approach to the interpretation of sub-Ångstrom resolution electron microscope images and microanalysis. Ultramicroscopy 94, 309-327.

Cowley, J.M. (1988). Electron microscopy of crystals with timedependent perturbations. Acta Cryst A 44, 847-853.
Cowley, J.M. (1995). Diffraction Physics (3rd ed.). Amsterdam, The Netherlands: Elsevier Science.

Dinges, C., Berger, A. \& Rose, H. (1995). Simulation of TEM images considering phonon and electronic excitations. Ultramicroscopy 60, 49-70.

Howie, A. (1979). Image contrast and localized signal selection techniques. J Microsc 117, 11-24.

Loane, R.F., Xu, P. \& Silcox, J. (1992). Incoherent imaging of zone axis crystals with ADF STEM. Ultramicroscopy 40, 121-138.

Pennycook, S.J. \& Jesson, D.E. (1991). High-resolution Z-contrast imaging of crystals. Ultramicroscopy 37, 14-38.

Rafferty, B., Nellist, P.D. \& Pennycook, S.J. (2001). On the origin of transverse incoherence in Z-contrast STEM. J Electron Microsc 50, 227-233.

WANG, Z.L. (1995). Dynamical theories of dark-field imaging using diffusely scattered electrons in STEM and TEM. Acta Cryst A 52, 569-585.

WANG, Z.L. \& Cowley, J.M. (1989). Simulating high-angle annular dark-field STEM images including thermal diffuse scattering. Ultramicroscopy 31, 437-454. 
8 1 C.

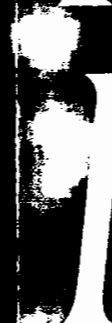

1

$+3$

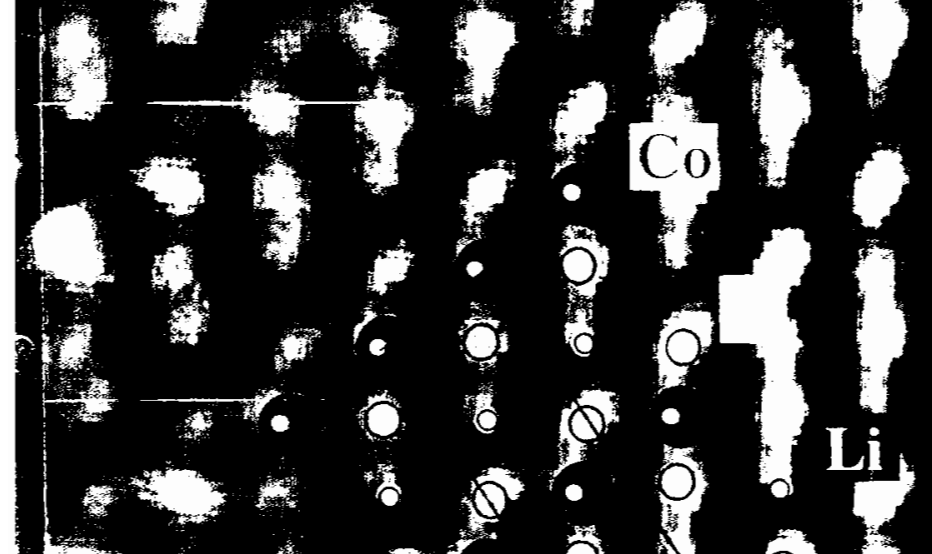

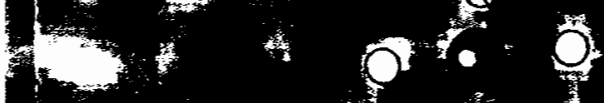
$\ln ^{2}, 0$

.

.

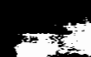

1

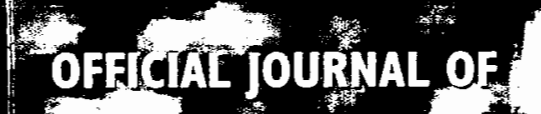

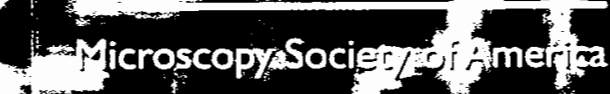

Microbeam Aneil sis Sérjety

Microscopial Socjety of Capada

Mexiean Tižcroscopy Societ /

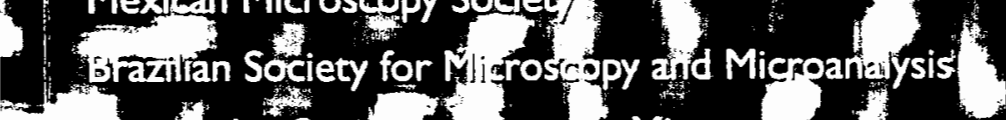
- venetidelan Socie of flectron Microscapy

(a)

Q

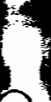

P.

Q)

i

0

7

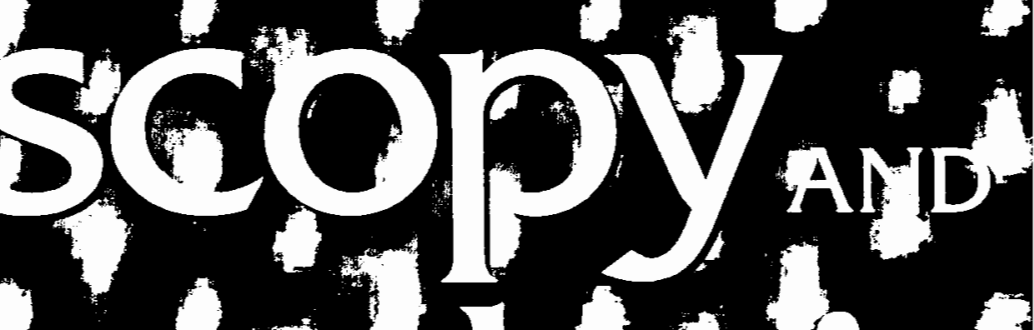

Vot he 10, Noimist

Fet ary' 2004

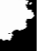


ARIZONA STATE UNIVERSITY

Center for High Resolution Electron Microscopy

Center for Solid State Science

Tempe, Arizona 85287-1704
Phone: (US) (480)-965-4540

Internet: david.smith@ asu.edu

Fax: (US) (480)-965-9004

Memorandum

To: Goff Ansis

From: Dave Smith

Re: Acceptance of Paper

Date: 24 April 03.

Your paper will be accepted but you should consider the attached referees' remarks in making some minor revisions. And maybe there were some errors introduced by my software so methinks yon should me a. hard copy lin duplicate) of the final version. Please also make the necessary "editorial" corrections as shown.

best wishes

Dave $S$ 


\section{Review of}

The 1s-state analysis applied to high-angle, annular dark-field image interpretation - when can we use it?

By Geoffrey R. Anstis.

This paper deals with a topic of current interest, since the interpretation of HAADF STEM images, particularly of semiconductors and their interfaces, is highly relevant for nanotechnology, and the applications of the new aberrationcorrected STEM instruments for such studies are being explored. There are several schools of thought on this and their ideas seem to be converging. This paper assists with the convergence. The conclusion is that for the aberration-corrected instruments the $1 \mathrm{~s}$ state picture should be a good approximation.

This paper follows on from the recent paper by Anstis, Cai and Cockayne in Ultramicroscopy (2003) and extends and reinforces those results, with calculations for InAs, to add to those for diamond and GaAs. It is a useful contribution to the literature and warrants publication.

The weakness of the paper is in the presentation.

In my copy, all the Greek letters are missing in the text, the figure captions and the Table. This makes it hard to read.

Also, there is too much reliance on knowledge of the previous publications of the author so that it is difficult to understand the argument without hunting up previous papers. For example, on the top of page 5 , what is the meaning of "scattering of the order $(\mathrm{s}, \mathrm{t})$ ".? What is the meaning of the notation"22/40" in Figs, 3 and 4? A few more words of explanation would help.

Recommendation: accept for publication after attention to these details. 
Re. "The 1s-state analysis applied to high-angle, annular dark-field image interpretation when can we use it", by Anstis

This paper reports dynamic calculations on the contrast contributed by TDS in HAADF STEM image. The paper addresses an important question whether the 1s-state is an excellent approximation; if yes, what are the conditions. It has long been interpreted that HAADF STEM image as the Z-contrast image and be simplified in a way that the image contrast is a sole representation of the projected atomic number along the column. This interpretation is valid in most of the cases for very thin samples, but for the cases of interfaces and dislocations cores, the Z-contrast approximation could be quite off the true structure. By pointing this out through theoretical calculation, the author has made his argument. The results will be useful for others to re-examine this long believed story. The paper is suggested for publication after minor revisions as specified below.

1. There are many symbols missing in the text and in figure caption, which may be due to the errors introduced by the software.

2. The following papers should be quoted in the references because they are closely related to the topics described. In fact, some of the points presented by the author have been discussed in these papers:

Z.L. Wang, "Dynamical Theories of Dark-Field Imaging Using Diffusely Scattered Electrons iIn STEM and TEM," Acta Crystal. A, 51 (1995), p. 569-585.

Z.L. Wang, "Electron Statistical Dynamical Diffuse Scattering in Crystals Containing Short-Range Order Point Defects," Acta Cryst. A52 (1996), p. 717-729. 


\section{Microscopy $_{\text {ano }}$ Microanalysis}

\section{Volume 10, Number I SPECIAL Issue IN Honor of Professor February 2004}

Acknowledgments

\section{INTRODUCTION}

An International Workshop in Honor of Regents' Professor John Maxwell Cowley on the Occasion of His 80th Birthday

David J. Smith

\section{Feature Articles}

The 1s-State Analysis Applied to High-Angle, Annular Dark-Field Image Interpretation-When Can We Use It?

Geoffrey R. Anstis

Off-Axis STEM or TEM Holography Combined with Four-Dimensional Diffraction Imaging

J.M. Cowley

The Precession Techinique in Electron Diffraction and Its Application to Structure Determination of Nano-Size Precipitates in Alloys

J. Gjønnes, V. Hansen, and A. Kverneland

Electron Microscopy of Biological Macromolecules: Bridging the Cap between What Physics Allows and What We Currently Can Get

Dieter Typke, Kenneth H. Downing, and Robert M. Glaeser

Ultralow-Energy Excitations and Prospects for Spatially Resolved Spectroscopy

A. Howie

FFT Multislice Method-The Silver Anniversary

Kazuo Ishizuka

Probing of Individual Semiconductor Nanowhiskers by TEM-STM

Magnus W. Larsson, L. Reine Wallenberg, Ann I. Persson, and Lars Samuelson

Screw Dislocations in GaN Grown by Different Methods

Z. Liliental-Weber, D. Zakharov, J. Jasinski, M.A. O'Keefe, and H. Morkoc

Advanced Electron Microscopy Characterization of Nanostructured Heterogeneous Catalysts

On Cover: Angstrom image of 'C.". thium baitery material at $\mathbf{5 0}$ in: nes magnification. This is the ixst $\mathrm{T}$ E.Y image of $\mathrm{Li}$ atoms. It was reconstructed from 20 experimental images. Inset simulation (right) is for a specimen of $48 \AA$ thickness at $0.9 \AA$ resolution. See Figure 9 in the article by Jingyue Liu

In Situ Electron Microscopy Studies of the Sintering of Palladium Nanoparticles on Alumina during Catalyst Regeneration Processes

Rou-Jane Liu, Peter A. Crozier, C. Michael Smith, Dennis A. Hucul, John Blackson, and Ghaleb Salaita 
Sub-Angstrom Atomic-Resolution Imaging from Heavy Atoms to Llght Atoms Michael A. O'Keefe and Yang Shao-Horn

Quantitative Analyses of Precession Diffraction Data for a Large Cell Oxide

Christopher S. Own, Arun K. Subramanian, and Laurence D. Marks

In Situ Reflection Electron Microscopy of Ge Island Nucleation on Mesa Structures

F.M. Ross, M. Kammler, M.E. Walsh, and M.C. Reuter

Beam Statistics and Diffraction from Materlals in the Critical State

J.R. Sellar

Characterization of a Co-CoO Obliquely Evaporated Magnetic Tape by

Analytical Electron Microscopy and Electron Holography

Daisuke Shindo, Masasuke Hosokawa, Zheng Liu, Yasukazu Murakami, Takuya Ito, Yoh Iwasaki, and Junichi Tachibana

Observation of Nanometer-Xe Clusters Embedded in Al Crystals

M. Song, K. Mitsuishi, and K. Furuya

Convergent-Beam Low Energy Electron Diffraction (CBLEED) and the Measurement of Surface Dipole Layers

J.C.H. Spence, H.C. Poon, and D.K. Saldin

Direct UHV-TEM Observation of Palladium Clusters on a Sillcon Surface

Masaki Takeguchi, Kazutaka Mitsuishi, Miyoko Tanaka, and Kazuo Furuya

First Observation of $\ln _{x} \mathrm{Ga}_{1-x}$ As Quantum Dots in GaP by Spherical-AberrationCorrected HRTEM in Comparison with ADF-STEM and Conventional HRTEM Nubuo Tanaka, Jun Yamasaki, Shingo Fuchi, and Yoshikazu Takeda

Observation of Magnetic Multilayers by Electron Holography

T. Tanji, S. Hasebe, Y. Nakagami, K. Yamamoto, and M. Ichihashi

Physical Limits on Atomic Resolution

D. Van Dyck, S. Van Aert, and A.J. den Dekker

Properties of Nanobelts and Nanotubes Measured by in Situ TEM

Zhong Lin Wang

\section{Book Review}

Quick Photoshop for Research: A Guide to Digital Imaging for Photoshop 4x, $5 x, 6 x, 7 x$ by Jerry Sedgewick Joe Morre

\section{Calendar of Meetings and Courses}




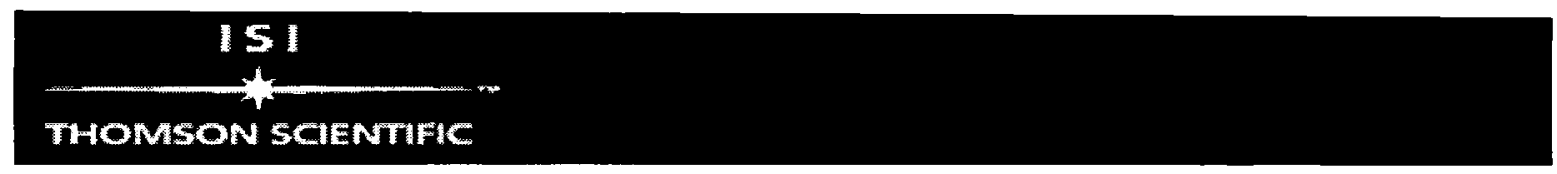

(Use Browser Back button to return to previous page)

\section{Thomson Scientific Master Journal List JOURNAL LIST}

Search terms: 1431-9276

Total journals found: 1

\section{MICROSCOPY AND MICROANALYSIS}

Bimonthly

ISSN: 1431-9276

CAMBRIDGE UNIV PRESS, 40 WEST 20TH ST, NEW YORK, USA, NY, 10011-4211 